\title{
Intuition in der Beratung
}

\author{
Silja Kotte
}

Online publiziert: 3 . November 2020

(C) Springer Fachmedien Wiesbaden GmbH, ein Teil von Springer Nature 2020

Der Begriff Intuition wird in einer Vielzahl unterschiedlicher Bedeutungen verwendet, die einander teilweise sogar widersprechen: Intuition wird als Gefühlsentscheidung verstanden, als Geistesblitz oder Eingebung, als die mittels einer Inkubationszeit unbewussten Verarbeitens gereifte Einsicht (,eine Nacht drüber schlafen“), als geronnene Erfahrung, als ein Wiedererkennen (Kahneman 2012, S. 237), als Einfühlungsvermögen, aber auch als fehleranfällige heuristische Form der Informationsverarbeitung, als Ausflucht vor einer vertieften, rationalen Auseinandersetzung und als Legitimation dafür, sich der intersubjektiven Nachvollziehbarkeit und einer Begründung des eigenen Entscheidens und Handelns zu entziehen.

Auch in der wissenschaftlichen Literatur gibt es viel Uneinigkeit, was genau unter Intuition zu verstehen sei, gerade weil das Forschungsfeld interdisziplinär ausgerichtet ist. Herauskristallisiert hat sich als gemeinsamer Nenner jedoch, dass unter Intuition in der Regel eine nicht bewusste, ganzheitliche, assoziative, affektiv geladene, oft schnelle Art und Weise, Informationen zu verarbeiten und zu Schlussfolgerungen zu kommen, verstanden wird (Dane und Pratt 2009; Hodgkinson et al. 2008; Mavor et al. 2010). Angesichts der mit der Intuition verbundenen Chancen und Risiken kommt zunehmend in den Blick, wovon die Qualität intuitiver Entscheidungen abhängt: Der Komplexitätsgrad der Entscheidung etwa spielt eine Rolle, genauso wie der Grad an Expertise und Erfahrung der Entscheider/innen, ihr Stresslevel und die Umgebungsbedingungen.

Intuitive Prozesse sind allgegenwärtig, sowohl im Arbeits- als auch im Privatleben (Mavor et al. 2010) und damit auch in Beratungsformaten wie Coaching, Supervision und Organisationsberatung. In der Praxisliteratur wird der Intuition oft eine

Prof. Dr. S. Kotte $(\bowtie)$

Kommunikation und Wirtschaft, HMKW Hochschule für Medien,

Solmsstraße 18, 60486 Frankfurt/M., Deutschland

E-Mail: s.kotte@hmkw.de 
wichtige Rolle im Coaching zugeschrieben, und einige Coachingverbände, wie etwa die International Coach Federation, haben die Intuition in ihre Kompetenzstandards aufgenommen. Aus einer wissenschaftlichen Perspektive wissen wir hingegen noch sehr wenig darüber, welche Rolle die Intuition im Coaching spielt, ob und wie Coaches mit ihrer Intuition arbeiten, was passiert, wenn ihre Intuition sie fehlleitet, sie die Intuition zurückhalten, ihr ausweichen oder sie als Grundlage für Hypothesenbildung und Intervention nutzen oder wie sie ,,the potential in the moment between [...] noticing and responding to an intuition“" (Sheldon 2018, S. 6) nutzen.

Die Beiträge zum Themenschwerpunkt Intuition gehen der Rolle der Intuition in der Beratung, in verwandten Feldern wie der Personalauswahl und in gesellschaftlich-politischen Prozessen aus verschiedenen Perspektiven nach: aus theoretischen und empirischen, aus psychologischen und soziologischen, aus wohlwollenderen und kritischeren Perspektiven. Zusammen sollen sie zum Nachdenken über das eigene Verständnis von Intuition, ihre Chancen und Risiken und ihre Rolle im gesellschaftlichen Kontext, in den Beratung eingebettet ist, anregen.

Der Beitrag von Eva Jonas, Anna Moser und Markus Quirin beschreibt, wie Intuition auf der Grundlage psychologischer Forschung und Theoriebildung verstanden werden kann. Die Autor/innen erläutern die Zwei-Prozess-Modelle der Informationsverarbeitung, die zwischen bewusst-analytischen und unbewusst-assoziativen Prozessen unterscheiden und innerhalb derer die Intuition den unbewusst-assoziativen Prozessen zugeordnet wird. Auf Grundlage der Theorie der PersönlichkeitsSystem-Interaktionen (PSI), schlagen sie ein differenzierteres Verständnis von Intuition vor, indem sie eine niedere und eine höhere Form von Intuition unterscheiden. Den Bogen zur Beratungspraxis schlagen sie über den Umgang mit der hohen Komplexität von Beratungssituationen im Spannungsfeld zwischen Reflexion und Intuition.

Silja Kotte, Alessa Müller und Isabell Diermann beschäftigen sich mit der Rolle der Intuition in der Eingangsdiagnostik im Coaching. Sie stellen die Ergebnisse zweier empirischer Untersuchungen vor. Auf Basis einer qualitativen Interviewstudie identifizieren sie fünf „Intuitions-Typen“ unter den Coaches sowie Strategien, die Coaches einsetzen, damit ihre Intuition sie nicht fehlleitet, sondern möglichst zielführend für den Prozess nutzbar wird. Die quantitativen Ergebnisse zeigen, auf welche Weise diejenigen Coaches, die angeben, die Intuition häufig zu nutzen, in der Eingangsdiagnostik vorgehen und wie Einsatz und Bewertung der Intuition mit Erfahrung zusammenhängen.

Sabrina Kohlmann geht genauer auf die Rolle von Erfahrung ein und untersucht den Zusammenhang von Expertise und bewusstem vs. intuitiv-unbewusstem Denken. Als Ausgangspunkt nimmt sie die Theorie des unbewussten Denkens, die davon ausgeht, dass Ablenkungsphasen, die es erlauben, das Unbewusste ,arbeiten“ zu lassen, unbewusst-intuitiv nachzudenken und dann erst zu entscheiden, in komplexen Situationen zu besseren Entscheidungen führen können. Die Ergebnisse eines Feldexperiments in der Altenpflege zeigen, dass unbewusstes Denken bei Expert/innen, nicht aber bei Noviz/innen zu besseren Personalauswahlentscheidungen führt. In der Übertragung auf die Beratungspraxis gibt die Autorin u.a. Anregungen dafür, wie Ablenkungsphasen, die es erlauben das Unbewusste ,,arbeiten“ zu lassen, in der Beratung aussehen können. 
Im Diskurs-Beitrag nimmt Helmut Willke eine bewusst kritische Haltung gegenüber der Intuition ein. Im historischen Überblick zeigt er auf, wie Entscheidungstheorien im Spannungsfeld angenommener Entscheidungsfreiheit vs. Determinismus stehen, und beschreibt Intuition als Mechanismus, wie Menschen in hochkomplexen und intransparenten Konstellationen dennoch überhaupt entscheiden können. Er verdeutlicht, welche problematischen, auch politisch-gesellschaftlichen Konsequenzen sich ergeben, wenn Intuition zur Flucht aus der Komplexität und aus der Rationalität genutzt wird, und plädiert für Intuition als eine hybride Form des Entscheidens auf der Grundlage begrenzter Rationalität einerseits und der expliziten Nutzung impliziten Wissens andererseits.

Neben diesen Beiträgen zum Themenschwerpunktfinden sich drei weitere Hauptbeiträge in diesem Heft. Ihre Themen weisen jeweils Bezüge zur klinischen Psychologie und Psychotherapie auf:

Johannes Ehrenthal, Heidi Möller und Jannik Zimmermann beschäftigen sich mit Brüchen in der Arbeitsbeziehung in Coaching und Supervision. Ausgehend davon, dass die Arbeitsbeziehung ein zentraler Wirkfaktor in Coaching und Supervision, aber auch anfällig für Störungen ist, stellen sie das aus der Psychotherapieforschung stammende Konzept der Brüche der Arbeitsbeziehung und ihrer „Reparatur“ vor. Sie geben Einblicke in das Konzept, u. a. in die unterschiedlichen Arten von Brüchen, und übertragen dieses auf die arbeitsweltliche Beratung.

Herbert Csef setzt sich mit dem Thema Derailment und Führungsversagen speziell bei weiblichen Führungskräften auseinander. Er geht insbesondere auf das Konzept und empirische Befunde zur dunklen Triade ein, d.h. zur Kombination aus Narzissmus, Machiavellismus und Psychopathie. Die Überlegungen überträgt er dann auf fünf Beispiele prominenter weiblicher Führungskräfte, deren Derailment öffentlich bekannt wurde und in der medialen Berichterstattung prominent war: die Betrügerin und Hochstaplerin Elizabeth Holmes sowie vier weitere Führungskräfte, die an der Spitze von Forschungsinstitutionen standen bzw. stehen.

Der Beitrag von Sandra Diller, Camilla Stadlinger, Irina Eberhard und Eva Jonas fokussiert auf den Narzissmus als Teilaspekt der dunklen Triade. Sie arbeiten heraus, wie sich narzisstische Tendenzen im Arbeitskontext und innerhalb von Coachingprozessen zeigen und inwiefern narzisstische Klient/innen von Coaches als schwierig erlebt werden und zu Angst und Disstress führen. Im Ausblick verweisen die Autorinnen auf Achtsamkeitsübungen als mögliche Strategie zum Umgang mit narzisstischen Klient/innen.

Die beiden Praxisberichte in diesem Heft widmen sich unterschiedlichen Themen: In ihrem Beitrag zu Supervision im Feld der Hospiz- und Palliativversorgung führt Ursula Hermann zunächst in das Feld von Palliative Care ein. Als Themenbereiche, die in der Supervision in diesem Feld besonders wichtig sind, identifiziert sie die widersprüchliche Zuweisungspraxis von Gesundheitsorganisationen zum Angebot Palliative Care und den Umgang mit dem Thema Abschied.

Im Beitrag zum Thema Design Thinking setzt sich Ernestine Feustel-Liess mit der Frage auseinander, welche Hürden des Innovationstransfers sich im Anschluss an Design Thinking Workshops ergeben und wie diese so gestaltet werden können, dass die erarbeiteten Innovationen auch implementiert werden. Auf der Grundlage 
einer Fallstudie in einem Unternehmen entwickelt sie Ansätze, wie der Bogen zu einer weiterführenden Innovationsberatung geschlagen werden kann.

Die Rubrik Diskurs enthält ein Interview, das Heidi Möller zum Thema „Künstler in Corona-Zeiten“ mit Alina Rank, einer freien Schauspielerin, und Jan Freese, einem freien Bühnenbildner, geführt hat: Wie verarbeiten Menschen, die von einem Tag auf den anderen ohne Arbeit dastehen, diese Krise? Wie sieht ihr inneres Erleben aus? Welche Antworten finden sie auf die Herausforderungen der Pandemie? Dieser Beitrag ist Teil des „Corona-Streams“, einer Reihe von Beiträgen in den nächsten Heften und vorab als Online-first-Veröffentlichungen, in denen die Folgen der Pandemie für Organisationsberatung, Supervision und Coaching thematisiert werden.

\section{Literatur}

Dane, E., \& Pratt, M. G. (2009). Conceptualising and measuring intuition: a review of recent trends. International Review of Industrial and Organisational Psychology, 24, 1-40.

Hodgkinson, G. P., Langan-Fox, J., \& Sadler-Smith, E. (2008). Intuition: a fundamental bridging construct in the behavioural sciences. British Journal of Psychology, 99, 1-27.

Kahneman, D. (2012). Thinking, fast and slow. London: Penguin Books.

Mavor, P., Sadler-Smith, E., \& Gray, D.E. (2010). Teaching and learning intuition: some implications for HRD and coaching practice. Journal of European Industrial Training, 34(8/9), 822-838.

Sheldon, C. (2018). Trust your gut, listen to reason: How experienced coaches work with intuition in their practice. International Coaching Psychology Review, 13(1), 6-20. 